\title{
Characterization of phenanthrenequinone-doped poly(methyl methacrylate) for holographic memory
}

\author{
Gregory J. Steckman, Iouri Solomatine, Gan Zhou, and Demetri Psaltis \\ Department of Electrical Engineering, California Institute of Technology, Mail Stop 136-93, Pasadena, California 91125 \\ Received May 22, 1998 \\ The holographic recording characteristics of phenanthrenequinone- (PQ-) doped poly(methyl methacrylate) \\ are investigated. The exposure sensitivity is characterized for single-hologram recording, and the $M / \#$ \\ is measured for samples as thick as $3 \mathrm{~mm}$. Optically induced birefringence is observed in this material. \\ (C) 1998 Optical Society of America \\ OCIS codes: $\quad 090.2900,090.4220,210.2860$
}

The characterization of phenanthrenequinone- (PQ-) doped poly(methyl methacrylate) $)^{1,2}$ (PMMA) as a recording material for holographic memories is described in this Letter. This material consists of the polymer host matrix with added $\mathrm{PQ}$ molecules as photosensitive dopant. High-optical-quality samples of this material were made with variable thicknesses of up to $5 \mathrm{~mm}$ and in a variety of shapes. This material does not shrink after exposure and is lightweight, inexpensive, and durable, making it an attractive candidate for disk-based holographic memory systems.

Sample preparation consists of dissolving PQ molecules in liquid methyl methacrylate together with a polymerization initiator. This solution is then poured into molds and allowed to polymerize in a pressure chamber at an elevated temperature. The molding process allows samples to be fabricated in a variety of geometries. Disks ranging from 2.5 to $10 \mathrm{~cm}$ in diameter with $1-5-\mathrm{mm}$ thickness were made. For a 1-mm-thick sample doped with a concentration of $0.7 \%$ of $\mathrm{PQ}$ molecules before exposure, the absorption reaches a maximum of $98.8 \%$ at $445 \mathrm{~nm}$ and is $58 \%$ for the 488-nm line of an argon laser, which was used in all experiments described in this Letter.

A hologram was recorded by a pair of 488-nm beams, each incident upon the material at an outside angle of $21.5^{\circ}$. We monitored the growth of the hologram during recording by probing the sample with a Bragg-matched $\mathrm{He}-\mathrm{Ne}$ laser beam. Figure 1 shows the diffraction efficiency (diffracted power divided by the incident power) during recording in 1-mm-thick material. The diffraction efficiency reached a maximum of $4.3 \%$ for an exposure energy of $2.5 \mathrm{~J} / \mathrm{cm}^{2}$. If exposure was allowed to continue, the diffraction efficiency began to drop. After $20 \mathrm{~J} / \mathrm{cm}^{2}$ of exposure with a single beam the hologram decayed to approximately $0.1 \%$. At this point the material was completely exposed, and no more holograms could be recorded.

We recorded permanent holograms that do not decay with subsequent illumination by stopping the exposure before saturation was reached and then baking the sample. Figure 2 shows the strength of a hologram as a function of baking time at a temperature of $55^{\circ} \mathrm{C}$. The diffraction efficiency reached a maximum after 12 days and remained steady with continued baking.

0146-9592/98/161310-03\$15.00/0
Figure 3 shows the selectivity curves for a weak and a strong hologram (2\% and $35 \%$ diffraction efficiency, respectively). The $2 \%$ hologram has a sinc-squared selectivity curve as expected for a 1-mm-thick hologram. The stronger hologram, on the other hand, has a selectivity curve that is distorted and shifted. For a holographic memory the diffraction efficiency is relatively small because many holograms are multiplexed. Therefore this effect is not going to be observed in practice. For characterization purposes the diffraction efficiency shown in Figs. 2 and 4 is calculated as the peak of the ideal Bragg selectivity curve with the same area as that of the measured curve. We attribute the slight shift of the weak hologram to a repositioning error arising from the removal of the material from the optical setup for baking. Shrinkage is not a suspect because we were able to reconstruct high-bandwidth holograms completely.

The recording sensitivity was measured for 1- and 3-mm-thick samples as a function of exposure energy and is shown in Fig. 4. For each exposure energy we recorded two holograms, each in a different sample of recording material, and then baked each sample for $48 \mathrm{~h}$ at $55^{\circ} \mathrm{C}$ to partially reveal the holograms. Plotted in Fig. 4 is the average of the equivalent diffraction efficiencies of the two trials with their standard deviation used as the error. For 1-mm-thick samples the maximum diffraction efficiency was achieved at exposure energy of $0.9 \mathrm{~J} / \mathrm{cm}^{2}$. For

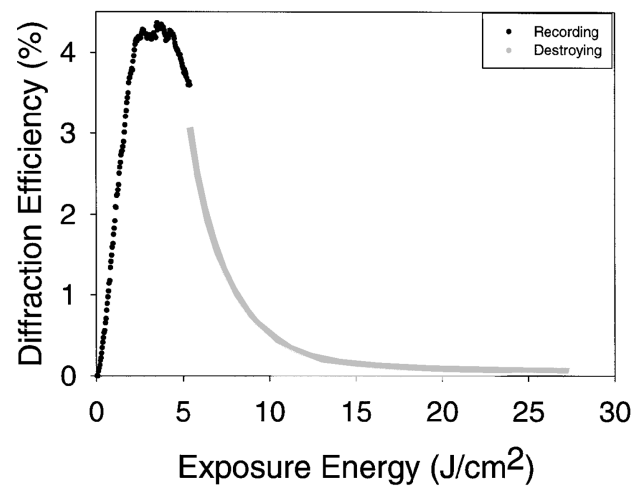

Fig. 1. Diffraction efficiency versus exposure energy as the hologram is being recorded.

(C) 1998 Optical Society of America 


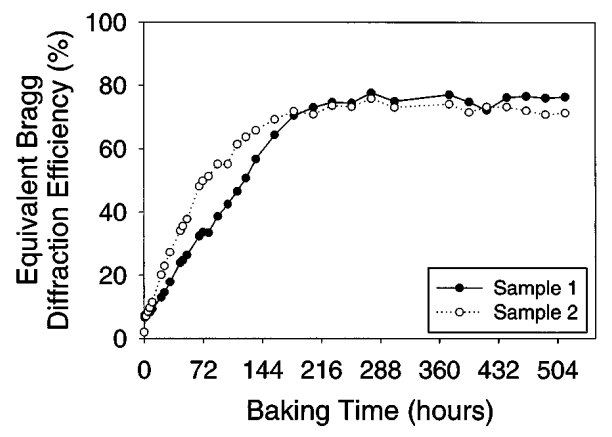

Fig. 2. Diffraction efficiency versus baking time for a hologram exposed to $1 \mathrm{~J} / \mathrm{cm}^{2}$ of energy.
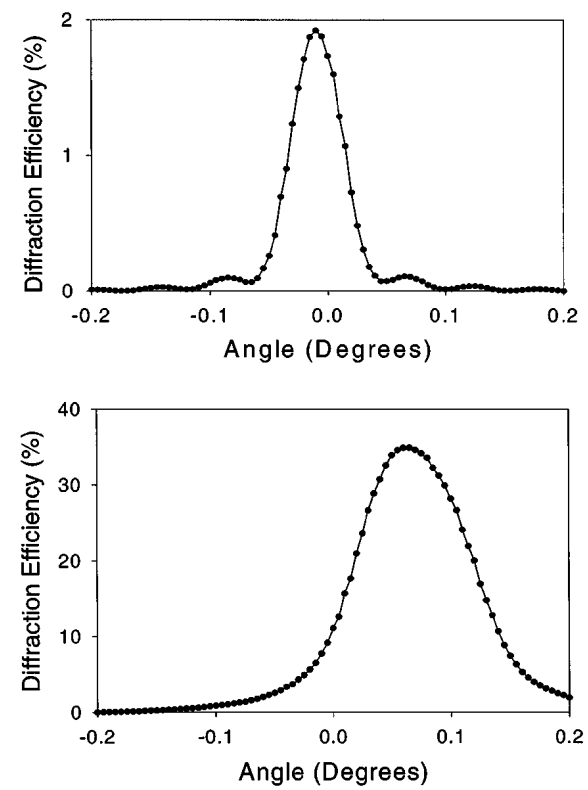

Fig. 3. Selectivity curves for a 1-mm-thick sample for a weak (top) and a strong (bottom) hologram.

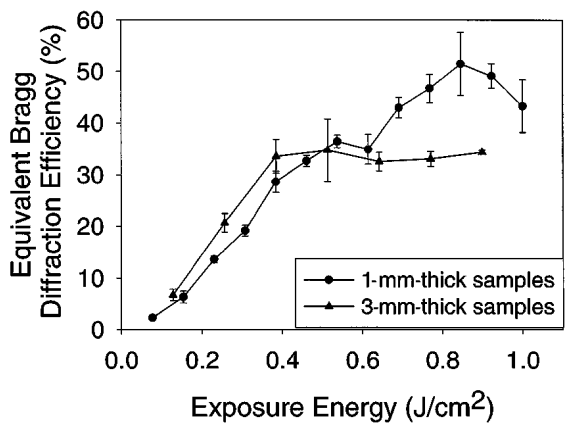

Fig. 4. Exposure sensitivity of 1- and 3-mm-thick samples.

3-mm-thick samples the recording saturated after only $0.4 \mathrm{~J} / \mathrm{cm}^{2}$. In a separate experiment several holograms were recorded with varying beam intensities while the exposure energy was kept constant. Over the range of 2 to $40 \mathrm{~mW} / \mathrm{cm}^{2}$ the material's recording sensitivity is not strongly dependent on the intensities of the recording beams.

Subsequent exposure causes erasure of previously recorded holograms as in photorefractives; however, repeated exposure causes the material to saturate as in many other photopolymers. To design a strategy for recording multiple holograms in this materials, we must briefly discuss the physical mechanism involved. On illumination the PQ molecules are photoexcited and bond to the host PMMA matrix. When the molecules are illuminated with two interfering beams, this bonding occurs primarily in regions of constructive interference. Through this process two partially offsetting gratings are created, one consisting of photoexcited $\mathrm{PQ}$ molecules that have bonded to the host polymer and the other of $\mathrm{PQ}$ molecules that are not bonded to the host polymer. At room temperature there is minimal diffusion of the $\mathrm{PQ}$ molecules through the polymer matrix. During heating, the free PQ molecules diffuse and distribute evenly, revealing the hologram. The diffusion time depends on both the grating frequency and the temperature. ${ }^{1}$ A final uniform exposure causes all the remaining free $\mathrm{PQ}$ molecules to bond to the PMMA matrix, thereby preventing any further holographic recording from taking place in the material. We also observed that the exposure of the material to uniform polarized light induces birefringence, with one of the optical axes aligned with the illuminating polarization. This effect was demonstrated experimentally and is shown in Fig. 5.

We recorded with equal exposure energy 50 planewave holograms at a single location of 3 -mm-thick samples, using peristrophic multiplexing. ${ }^{3}$ We summed the square root of the measured diffraction efficiencies to form the cumulative grating strength, ${ }^{4}$ defined as $C=\sum_{i=1}^{M} \sqrt{\eta_{i}}$. We then varied the cumulative exposure energy by increasing the exposure time of the individual holograms. Figure 6 shows the strengths of the 50 holograms recorded in a single sample. The cumulative grating strength with the 50 holograms recorded with different total exposure energies for different samples is shown in Fig. 7. The saturation value of the cumulative grating strength is

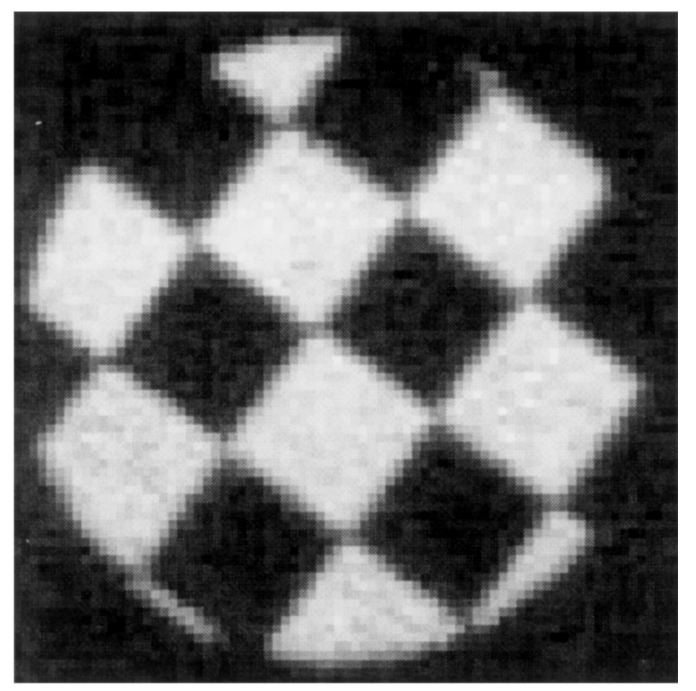

Fig. 5. 1-mm-thick sample illuminated with a checkerboard pattern of polarized light and imaged between two crossed polarizers. The illuminated regions exhibit birefringence. 


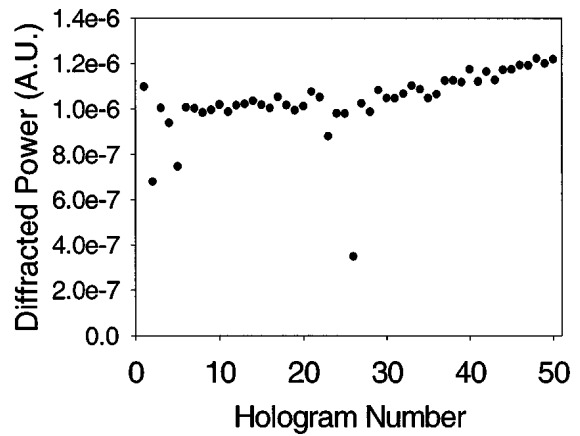

Fig. 6. Strength of 50 holograms recorded in a 3-mm-thick sample.

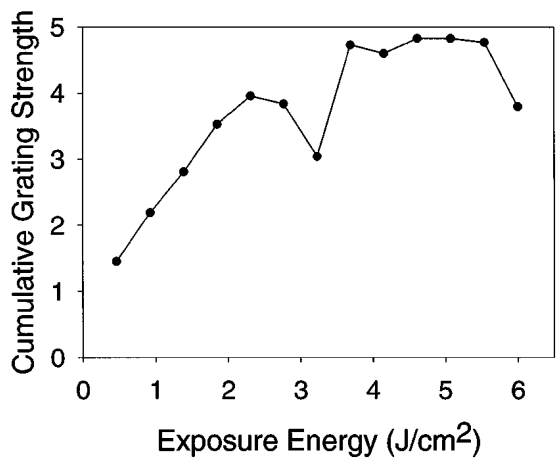

Fig. 7. Cumulative grating strengths for 50 holograms in 3 -mm-thick samples with varying exposure energy.

approximately equal to the $M / \#$ of the material. ${ }^{5}$ The $M / \#$ reaches a maximum of $M / 4.8$ for a cumulative exposure energy of $\sim 5 \mathrm{~J} / \mathrm{cm}^{2}$. With increased ex- posure energy the cumulative grating strength then begins to drop as a result of the hologram-destruction process. The earlier recorded holograms are reduced in strength by the recording of subsequent holograms, and the increase gained when we record for longer exposure energies is overpowered.

We conclude by comparing the properties of $\mathrm{PQ}$ doped PMMA with those of DuPont HRF-150 films, which have been used extensively for holographic storage. ${ }^{3,4}$ High-optical-quality, thick (5-mm) samples without shrinkage were made with $\mathrm{PQ}$ PMMA. The HRF-150 film had $M / 6.5$ for a $100-\mu$ m-thickness in Ref. 4 , whereas a value of $M / 4.8$ is reported here for a 3-mm-thick PQ PMMA sample. The main disadvantages of this material vis-a-vis the HRF-150 photopolymer are low sensitivity, $3 \mathrm{~J} / \mathrm{cm}^{2}$ in PQ-PMMA versus less than $300 \mathrm{~J} / \mathrm{cm}^{2}$ in HRF-150, and the baking requirement.

This work was supported by TRW as part of the Neuromorphic Systems Engineering Research Center, Rome Labs, and by the U.S. Air Force Office of Scientific Research. We thank Salim Shahriar for introducing us to this material.

\section{References}

1. A. V. Veniaminov, V. F. Goncharov, and A. P. Popov, Opt. Spektrosk. 70, 864 (1991).

2. V. I. Sukhanov, J. Opt. Technol. 61, 49 (1994).

3. K. Curtis, A. Pu, and D. Psaltis, Opt. Lett. 19, 993 (1994).

4. A. Pu, K. Curtis, and D. Psaltis, Opt. Eng. 35, 2824 (1996).

5. D. Psaltis, D. Brady, and K. Wagner, Appl. Opt. 27, 1752 (1988). 